\title{
Aberrant expression of long non-coding RNAs in peripheral blood mononuclear cells isolated from patients with gouty arthritis
}

\author{
XIAOWU ZHONG ${ }^{1-3^{*}}$, YUANHONG PENG ${ }^{4 *}$, HEBIN LIAO $^{2 *}$, CHENGJIAO YAO ${ }^{4}$, JIULONG LI $^{1,3}$, \\ QIBIN YANG ${ }^{4}$, YONGLONG HE ${ }^{4}$, YUFENG QING ${ }^{4}$, XIAOLAN GUO $^{1-3}$ and JINGGUO ZHOU ${ }^{5}$ \\ ${ }^{1}$ Department of Clinical Laboratory, Affiliated Hospital of North Sichuan Medical College, Nanchong, Sichuan 637000; \\ ${ }^{2}$ Translational Medicine Research Center, North Sichuan Medical College, Nanchong, Sichuan 637007; \\ ${ }^{3}$ Department of Laboratory Medicine, North Sichuan Medical College; ${ }^{4}$ Department of Rheumatology and Immunology, \\ Affiliated Hospital of North Sichuan Medical College, Nanchong, Sichuan 637000; ${ }^{5}$ Department of Rheumatology and \\ Immunology, The First Affiliated Hospital of Chengdu Medical College, Chengdu, Sichuan 610000, P.R. China
}

Received November 28, 2018; Accepted May 23, 2019

DOI: $10.3892 /$ etm.2019.7816

\begin{abstract}
Gouty arthritis (GA) is the most common inflammatory and immune-associated disease, and its prevalence and incidence exhibit yearly increases. The aim of the present study was to analyse the expression profile variation of long non-coding RNAs (lncRNAs) in GA patients and to explore the role of IncRNAs in the pathogenesis of GA. The peripheral blood mononuclear cells of GA patients and of healthy controls (HCs) were used to detect for the differentially expressed lncRNAs by microarray. The functional annotations and classifications of the differentially expressed transcripts were predicted using Gene Ontology (GO) and pathway analysis. The results were then verified by reverse transcription-quantitative (RT-q)PCR. A total of 1,815 lncRNAs and 971 mRNAs with a $>2$-fold difference in the levels of expression in the GA patients compared with those in the HCs were identified. According to the GO functional enrichment analysis, the differentially expressed lncRNAs were accumulated in terms including protein binding, catalytic activity and molecular transducer activity. The pathways predicted to be involved were the tumor necrosis factor signaling pathway, osteoclast differentiation, NOD-like receptor signaling pathway and NF- $\mathrm{kB}$ signaling pathway. The expression of six lncRNAs was measured by RT-qPCR and the results were consistent with those of the microarrays. Among these IncRNAs, AJ227913 was the most differentially expressed lncRNA in GA patients vs. HCs. The
\end{abstract}

Correspondence to: Professor Xiaolan Guo, Department of Clinical Laboratory, Affiliated Hospital of North Sichuan Medical College, 63 Wenhua Road, Nanchong, Sichuan 637000, P.R. China E-mail:alan5200@hotmail.com

*Contributed equally

Key words: gouty arthritis, peripheral blood mononuclear cells, long non-coding RNA, microarray expression of several lncRNAs was significantly changed in GA patients compared with that in HCs, which suggests that these IncRNAs with differential expression levels may have an important role in the development and progression of GA.

\section{Introduction}

Gouty arthritis (GA) is one of the most common types of inflammatory arthritis and is caused by the deposition of monosodium urate (MSU) crystals in and around joints. Elevated serum urate levels are recognized as an important risk factor for the development of GA (1). However, only $~ 10 \%$ of individuals with hyperuricaemia actually develop GA. Therefore, it has been suggested that hyperuricemia alone is not sufficient to cause GA (2). Although previous studies have identified several candidate risk genes (SLC2A9, ABCG2 and URAT1) associated with raised serum urate concentrations that increase the risk for an individual to develop GA (3-5), the exact pathogenesis of gout remains elusive. There may be other genes that are not associated with urate metabolism that also contribute to susceptibility to this disease.

Long non-coding RNAs (lncRNAs) are a group of RNA transcripts that are $>200 \mathrm{nt}$ in length. Initially, they were thought to be 'transcriptional noise' due to their lack of significant open reading frames and protein-coding ability. The human genome encoded tens of thousands of lncRNAs (6). In the past decade, an increasing number of IncRNAs have been characterized. Certain IncRNAs have been indicated to have important roles in diverse biological processes, including cell development, tumorigenesis and immune response. IncRNAs exert critical functions in the transcriptional, post-transcriptional and epigenetic regulation of gene expression (7). Although several studies have identified lncRNAs involved in a series of human biological and disease-associated processes $(8,9)$, only isolated examples on the regulation of rheumatic diseases through IncRNAs have been provided and the roles of IncRNAs in the progression of GA have remained to be fully elucidated.

In the present study, IncRNA microarrays were performed to evaluate the global expression profile of IncRNAs in 
peripheral blood mononuclear cells (PBMCs) of GA patients. In a subsequent Bioinformatics analysis, the lncRNA functions were annotated by Gene Ontology (GO) analysis, pathway analysis and network analysis. Reverse transcription-quantitative (RT-q)PCR was used to validate several random lncRNAs that were upregulated and downregulated in the GA patients compared with those in the healthy controls (HCs). These results provide information for future studies on GA.

\section{Patients and methods}

Study population and ethical statement. All participants were recruited from the Department of Rheumatology of the Affiliated Hospital of North Sichuan Medical College (Nanchong, China) between February 2015 and July 2016. The study group consisted of $60 \mathrm{GA}$ patients and $60 \mathrm{HC}$ subjects. The diagnoses of GA were confirmed by a clinical endocrinology physician, according to the American College of Rheumatology classification criteria (10). The patients had not received any systemic anti-inflammatory treatments or drugs to control the production and elimination of uric acid prior to obtainment of the blood samples. According to the design of a matched case-control study, healthy subjects with no history of gout and without any systemic inflammatory disease were enrolled in the present study. All the participants were of Chinese Han descent. Blood samples from all participants were obtained in the morning following overnight fasting for at least $12 \mathrm{~h}$, and were collected in sterile, single-use, anticoagulant-coated tubes and immediately sent to the laboratory for genetic testing.

RNA isolation. Ficoll-Hypaque density gradient centrifugation was performed to isolate the PBMCs from total blood samples. Total RNA was extracted from PBMCs using TRIzol reagent (Invitrogen; Thermo Fisher Scientific, Inc.) according to the manufacturer's protocol. RNA quantity and quality were measured using a NanoDrop ND-2000c spectrophotometer (Thermo Fisher Scientific, Inc.) All of the RNA samples were stored at $-80^{\circ} \mathrm{C}$ until further use.

Microarray analysis. Samples from three GA patients and three $\mathrm{HC}$ subjects were used for the lncRNA microarray analysis on an Agilent SurePrint G3 Gene Expression Microarrays for Human (v3) platform (part no. G4851C; Agilent Technologies). The RNA integrity of these samples was assessed with an Agilent Bioanalyzer 2100 (Agilent Technologies). In this study, RNA integrity number (RIN) and $28 \mathrm{~S}$ to $18 \mathrm{~S}$ rRNA ratio (28S/18S) were used as a criterion for RNA quality. Only samples with $2,100 \mathrm{RIN} \geq 7.0$ and $28 \mathrm{~S} / 18 \mathrm{~S} \geq 0.7$ were used. Total RNA was amplified and labelled with a Low Input Quick Amp Labeling Kit, One-Color (Agilent Technologies), following the manufacturer's protocol. Labelled cRNA (pmol Cy3/ $\mu \mathrm{g}$ cRNA) was purified with an RNeasy mini kit (Qiagen $\mathrm{GmBH}$ ). Each slide was hybridized with $1.65 \mu \mathrm{g}$ Cy3-labelled cRNA using a Gene Expression Hybridization Kit (Agilent Technologies) in a hybridization oven (Agilent Technologies) according to the manufacturer's protocol. After $17 \mathrm{~h}$ of hybridization, slides were washed in staining dishes (Thermo Fisher Scientific, Inc.) with a Gene Expression Wash Buffer Kit (Agilent Technologies) following the manufacturer's protocol. Slides were scanned with an Agilent microarray scanner (Agilent Technologies) with default settings (dye channel: Green, scan resolution $=3 \mu \mathrm{m}$, 20 bit). Data were extracted with Feature Extraction software 10.7 (Agilent Technologies). Raw data were normalized with a Quantile algorithm, with Gene Spring Software 11.0 (Agilent Technologies). Differentially expressed lncRNAs and mRNAs between the two groups were identified when fold-change $\geq 2$.

'Cis' and 'Trans' analysis of lncRNAs. The cis role of lncRNA refers to the lncRNA acting on neighbouring target genes $(11,12)$. The protein-coding genes $10 \mathrm{kbp}$ upstream or downstream of the lncRNAs were screened as potential 'cis'-interacting genes. The trans predictions were made using blast to identify complementary or similar sequences. The complementary energy between the two sequences was calculated by using RNAplex. The genes with e $\leq-30$ were selected as potential 'trans'-interacting genes (13).

Gene Ontology (GO) enrichment and pathway analysis. GO analysis was used to identify functional terms in different categories enriched by the differentially expressed mRNAs (www.geneontology.org; release 2016-08-08) (14). The KEGG database (www.genome.jp/kegg; release 79, 2016/07) was used to determine the biological pathways that were enriched by the differentially expressed mRNAs (15).

$R T$-qPCR assay. The lncRNAs selected and the sequences of the primers that were used for qPCR are provided in Table I. GAPDH was used as the endogenous control. In brief, total RNA was extracted as described above and then reverse-transcribed into complementary (c)DNA using a PrimeScript ${ }^{\circledR}$ RT Reagent kit with gDNA Eraser (Perfect Real Time; Takara Bio Inc.) following the manufacturer's protocol. The differential expression of lncRNAs that were identified in the microarray analysis was measured by qPCR using SYBR Green assays (Takara Bio Inc.). The reactions were performed in a final volume of $20 \mu \mathrm{l}$ and included $10 \mu \mathrm{l}$ Power SYBR Green PCR Master Mix, $0.4 \mu 1$ ROX Dye, $0.5 \mu 1$ final working concentration of $10 \mathrm{pmol} / 1$ of each of the forward and reverse primers, $2 \mu \mathrm{l}$ cDNA and $6.6 \mu \mathrm{l}$ nuclease-free water. The reactions were performed using a QuantStudio ${ }^{\mathrm{TM}}$ 12K Flex Real-Time PCR System (Applied Biosystems; Thermo Fisher Scientific, Inc.). The thermocycling conditions for PCR were as follows: Pre-denaturation at $95^{\circ} \mathrm{C}$ for $10 \mathrm{~min}$, and amplification for 40 cycles of denaturation at $95^{\circ} \mathrm{C}$ for $15 \mathrm{sec}$ and elongation at $60^{\circ} \mathrm{C}$ for $1 \mathrm{~min}$. All the experiments were performed in duplicate and the mean value was used for further analysis. Relative lncRNA concentrations were calculated using the $2^{-\Delta \Delta \mathrm{Cq}}$ method (16).

Statistical analysis. Statistical analysis was performed using the SPSS 22.0 software package (IBM Corp.). To analyse differences in expression of individual lncRNAs or mRNAs in the microarray analysis, Student's t-tests were used. Regarding the results of the PCR analysis, the significance of differences in expression levels of lncRNAs were determined by the Kruskal-Wallis test or Mann-Whitney U-test. For statistical correlation, Spearman correlation coefficient was used. Fisher's exact test was used in the GO enrichment and KEGG pathway analyses. $\mathrm{P}<0.05$ was considered to indicate statistical significance. 
Table I. Primer sequences used for validation of lncRNAs by reverse transcription-quantitative PCR.

\begin{tabular}{lllr}
\hline lncRNA & \multicolumn{1}{c}{ Forward primer (5'-3') } & \multicolumn{1}{c}{ Reverse primer (5'-3') } \\
\hline AJ227913 & TTTTGCCAAGGAGTGCTAAAGA & AACCCTCTGCACCCAGTTTTC & 194 \\
AK001903 & CCAGCGGATATTTTGGTGTTTG & AGAAGCTATCAGGCGTTGCTG & 86 \\
ENSG00000239182 & TAGCACTGTTGCCTGAGCCA & GGAAGGAGCAGCCCACAGC & 95 \\
lnc-AP000769.1-1 & CAAGCAGAAGCAACAGGTCA & GAGCCAGGAAGATTGGAGAA \\
lnc-PCYOX1L-1:1 & GGAAAGGCAGTAATCAACTCCA & ACTCCACAATCCCCACAGC & 144 \\
lnc-CCDC64B-1:8 & ACCCCCACCCCAGGTCTTC & GCTGTGTCTCTGTCTTGGTCTCTT & 268 \\
GAPDH & ATCATCCCTGCCTCTACTG & AGTCAGAGGAGACCACCTG & 241
\end{tabular}

lncRNA, long non-coding RNA.

\section{Results}

Overview of the IncRNA and $m R N A$ profiles. The expression levels of the lncRNAs and mRNAs from three GA patients and paired control samples were analysed using lncRNA expression microarrays, which contained a total of 63,431 lncRNAs. All lncRNAs and mRNAs that were differentially expressed with a fold-change $\geq 2.0$ between the GA and HC groups are provided in Tables SI and SII. Analysis of the microarray data revealed that 1,815 $\mathrm{lncRNAs}$ and $971 \mathrm{mRNAs}$ were significantly differentially expressed in GA patients compared with those in the HC group. In total, there were 875 upregulated lncRNAs and 940 downregulated lncRNAs in GA patients compared with those in the HC group (Fig. 1A). A total of 465 mRNAs were upregulated and 506 mRNAs were downregulated in the GA patients compared with those in the HC group (Fig. 1B). Table II presents the 10 most upregulated and downregulated lncRNAs in the GA patients vs. HC group. Among these lncRNAs, AJ227913 (fold-change, 17.014) was the most upregulated lncRNA and 52094-1 (fold-change, 11.772) was the most downregulated lncRNA in the GA patients vs. HC group. Table III lists the 10 most upregulated and downregulated mRNAs. The most significantly upregulated mRNA was NM_005217 (fold-change, 18.208), and the most downregulated mRNA was NM_005353 (fold-change, 14.047) in the GA patients vs. HC group. Studies have suggested that several lncRNAs regulate their own transcription in 'cis', as well as that of nearby genes, by recruiting remodelling factors to local chromatin (11). Therefore, the chromosomal co-expression was then analysed. According to this analysis, the lncRNAs had 148 'cis' genes and 145 'trans' genes. The downregulated lncRNAs had 233 'cis' genes and 338 'trans' genes (detailed results are displayed in Tables SI and SII).

GO analysis. GO analysis was performed to identify the transcripts with terms that covered 3 domains (Fig. 2): Biological process, cellular component and molecular function. Fisher's exact test was employed to determine if there was more overlap between the differentially expressed list and the GO annotation list than what would be expected by chance $(\mathrm{P}<0.05$ is recommended). The most highly enriched GO terms in the category molecular function were identified as 'binding', 'catalytic activity' and 'molecular transducer activity'. The most highly enriched GO terms in the category cellular component were 'cell' and 'cell part'. In the category biological process, the most highly enriched GO term was 'cellular process'. 'Metabolic process', and 'immune system process' were also significantly enriched.

Pathway analysis. KEGG pathway analysis was performed for the differentially expressed transcripts. The pathway analysis indicated which biological pathways were significantly enriched for the differentially expressed transcripts. A total of 19 pathways corresponded to the differentially expressed transcripts (Table IV). The gene category 'tumor necrosis factor (TNF) signaling pathway' was the most enriched network, promoting cell survival and differentiation, as well as immune and inflammatory responses (17). Furthermore, 8 transcripts were significantly enriched in the pathway 'osteoclast differentiation', which is closely linked to bone development and repair (18). Among these pathways, the 'NOD-like receptor signaling pathway' and 'NF- $\mathrm{KB}$ signaling pathway' (Fig. 3) have been previously demonstrated to be involved in MSU-mediated inflammation through activation of Toll-like receptors/MyD88-dependent NF- $\mathrm{BB}$ signaling, NLR family pyrin domain containing 3 inflammasome and interleukin (IL)-1//MyD88-dependent IL-1 receptor signalling (19).

$R T-q P C R$ validation. To validate the results of the microarray, four of the upregulated lncRNAs and two of the downregulated lncRNAs were selected for the RT-qPCR analysis of 60 GA patients and $60 \mathrm{HC}$ subjects from a Han Chinese population. The results confirmed that the expression of AJ227913 $(\mathrm{P}<0.05)$, AK001903 $(\mathrm{P}<0.05)$, ENSG00000239182 $(\mathrm{P}<0.05)$ and lnc-AP000769.1-1 ( $\mathrm{P}<0.05)$ was significantly increased in gouty arthritis patients compared with that in healthy subjects, whereas the expression of lnc-PCYOX1L-1:1 $(\mathrm{P}<0.05)$ and lnc-CCDC64B-1:8 $(\mathrm{P}<0.05)$ was significantly decreased in GA patients compared with that in healthy subjects. The fold changes of the normalized levels for the six lncRNAs were $17.02,10.97,7.71,2.03,2.31$ and 2.05, respectively, in the gene chip analysis comparing the GA to the HC groups. Regarding the expression levels in the RT-qPCR analysis, these fold changes were $10.76,7.64,4.15,3.16,2.03$ and 2.50, respectively, in the GA group compared to those in the control group. Thus, the results of the RT-qPCR and the microarray data were consistent (Fig. 4). Among the lncRNAs analysed, AJ227913 had the largest difference in the expression between the two 
Table II. Top 10 significantly differentially expressed lncRNAs between gouty arthritis patients and healthy controls.

A, Upregulated lncRNAs

\begin{tabular}{llrr}
\hline lncRNA name & \multicolumn{1}{c}{ Source database } & Fold change \\
\hline AJ227913 & NONCODERv3 & 17.014 & P-value \\
AK001903 & NONCODERv3 & 10.971 & 0.035 \\
THC2686626 & Agilent_HUMAN_G3V2 & 9.332 & 0.016 \\
TCONS_00026568 & Agilent_HUMAN_G3V2 & 8.450 & 0.034 \\
lnc-COL20A1 & lncipedia2.1 & 7.833 & 0.008 \\
ENST00000604411.1 & gencode_v17 & 7.741 & 0.038 \\
lnc-AP000769.1-1 & lncipedia2.1 & 7.710 \\
TCONS_00026396 & Agilent_HUMAN_G3V2 & 7.607 & 0.020 \\
TCONS_00018683 & Agilent_HUMAN_G3V2 & 7.526 \\
TCONS_12_00003767 & Agilent_HUMAN_G3V2 & 6.914 \\
\hline
\end{tabular}

B, Downregulated lncRNAs

\begin{tabular}{llrr}
\hline lncRNA name & \multicolumn{1}{c}{ Source database } & Fold change & P-value \\
\hline $52094-1$ & UCSC & 11.772 & 0.001 \\
TCONS_12_00000650 & Agilent_HUMAN_G3V2 & 11.392 & 0.007 \\
CR616125 & NONCODERv3 & 9.367 & 0.012 \\
lnc-KCNT2 & lncipedia2.1 & 8.885 & 0.018 \\
BX538226 & NONCODERv3 & 8.794 & 0.001 \\
ENST00000430519 & Agilent_HUMAN_G3V2 & 8.410 & 0.025 \\
lnc-APTX-1 & lncipedia2.1 & 7.178 & 0.001 \\
TCONS_12_00007044 & UCSC & 7.000 \\
37475-1 & NONCODERv3 & 6.935 \\
AK056081 & Agilent_HUMAN_G3V2 & 6.894 \\
\hline
\end{tabular}

lncRNA, long non-coding RNA; UCSC, University of California Santa Cruz.
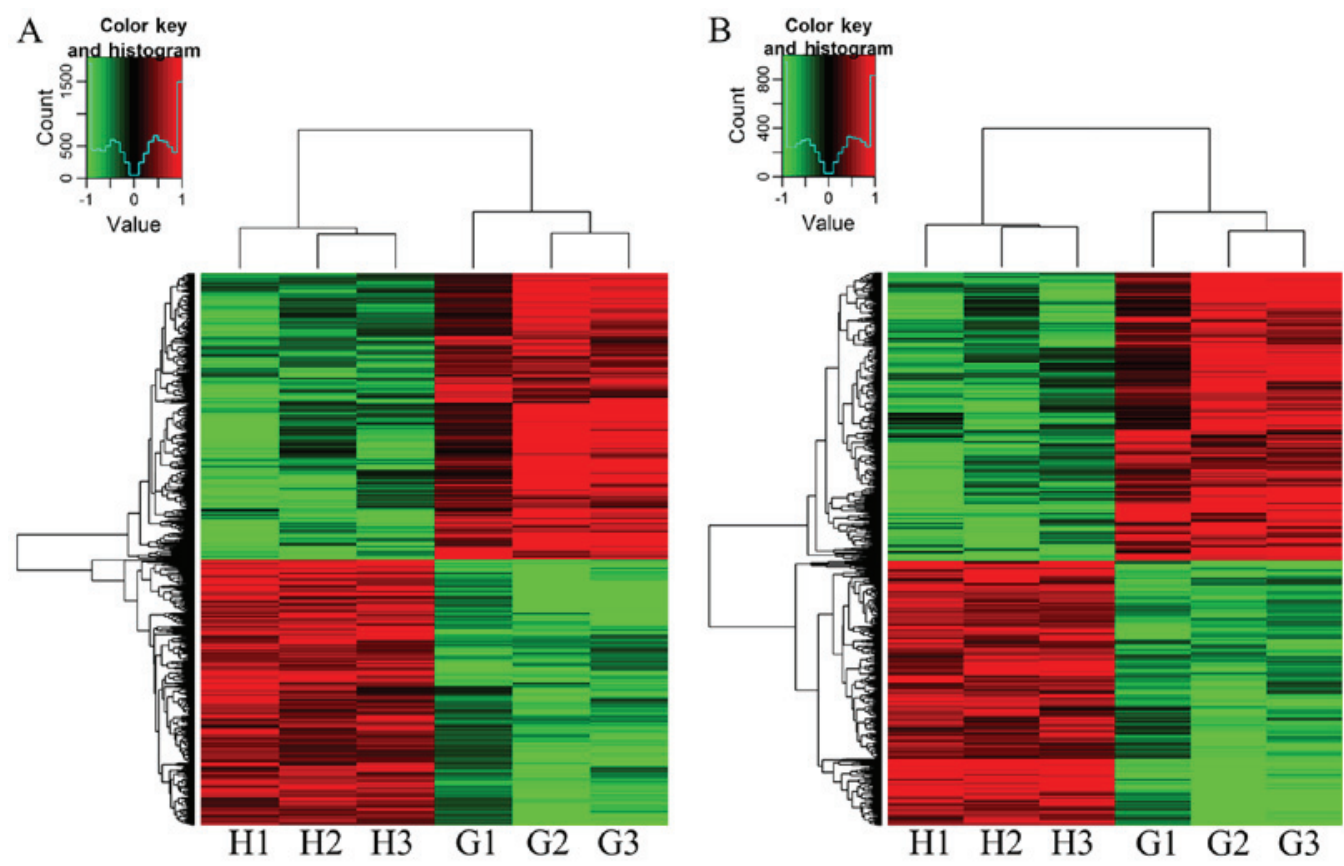

Figure 1. Expression profiling of (A) long non-coding RNAs and (B) mRNAs in GA patients and in healthy controls. Red and green colours represent up- and downregulated genes, respectively. G, GA patients; H, healthy controls. GA, gouty arthritis. 
Table III. Top 10 significantly differentially expressed mRNAs between gouty arthritis patients and healthy controls.

A, Upregulated mRNAs

\begin{tabular}{llll}
\hline Primary accession number & Gene symbol & Fold change & P-value \\
\hline NM_005217 & DEFA3 & 18.208 & 0.050 \\
NM_014364 & GAPDHS & 14.277 & 0.003 \\
NM_173557 & RNF152 & 11.779 & 0.032 \\
NM_000584 & IL8 & 11.616 & 0.030 \\
DB514319 & NA & 11.600 & 0.028 \\
NM_002192 & INHBA & 11.220 & 0.029 \\
THC2725968 & XLOC_002791 & 10.485 & 0.017 \\
ENST00000511330 & XLOC_003547 & 9.264 & 0.001 \\
NM_003955 & SOCS3 & 9.186 & 0.002 \\
NM_001964 & EGR1 & 8.8103 \\
\hline
\end{tabular}

B, Downregulated mRNAs

\begin{tabular}{llrr}
\hline Primary accession number & Gene symbol & Fold change & P-value \\
\hline NM_005353 & ITGAD & 14.047 & 0.007 \\
ENST00000435913 & NA & 8.365 & 0.016 \\
NM_015267 & CUX2 & 8.346 & 0.042 \\
NR_026970 & LY86-AS1 & 8.068 & 0.033 \\
ENST00000400449 & NA & 7.909 & 0.014 \\
AK095683 & PSMG4 & 7.880 & 0.037 \\
NM_021161 & KCNK10 & 7.879 & 0.022 \\
LNCA_33_P3384628 & NA & 7.670 & 0.010 \\
NM_080747 & KRT72 & 7.656 & 0.008 \\
NR_040093 & LOC100505678 & 7.343 & 0.016 \\
\hline
\end{tabular}

DEFA3, defensin, a 3; GAPDHS, glyceraldehyde-3-phosphate dehydrogenase, testis-specific; RNF152, ring finger protein 152; IL-8, interleukin 8; INHBA, inhibin $\beta$ A; SOCS, suppressor of cytokine signalling; EGR1, early growth response protein 1; CUX2, cut like homeobox 2; ITGAD, integrin $\alpha$-D; LY86-AS1, LY86 antisense RNA 1; PSMG4, proteasome assembly chaperone 4; KCNK10, potassium channel subfamily K member 10; KRT72, keratin, type II cytoskeletal 72; NA, not available.

groups. Furthermore, Spearman correlation analysis suggested that the expression levels of AJ227913 in GA patients were correlated with urea $(\mathrm{r}=0.618, \mathrm{P}<0.05)$, creatinine $(\mathrm{r}=0.382$, $\mathrm{P}<0.05)$ and cystatin $\mathrm{C}(\mathrm{r}=0.482, \mathrm{P}<0.05$; Table SIII).

\section{Discussion}

Protein-coding genes account for only 2\% of the human genome, while a large proportion of the genome is transcribed to generate non-coding RNAs that have been estimated to comprise up to $98-99 \%$ of the genome (20). It has become apparent that mutations and abnormal regulation of certain lncRNAs have important roles in the occurrence and development of various rheumatic diseases (21). Luo et al (22) indicated that 8,868 lncRNAs were highly differentially expressed in systemic lupus erythematosus (SLE) samples compared with those in a healthy group, and overall, aberrant expression profiles of IncRNAs may provide important insight into the pathogenesis of SLE (23). Downregulation of lnc-dendritic cell lncRNA (lnc-DC) in the plasma of patients with SLE may regulate type 17 T-helper cell differentiation by regulating the expression of signal transducer and activator of transcription 3; thus, the plasma levels of lnc-DC may be a potential biomarker for $\operatorname{SLE}(24,25)$. In rheumatoid arthritis (RA) patients, MALAT1 increases the expression of caspase- 3 and caspase- 9 and promotes cell growth and apoptosis of RA fibroblast-like synoviocyte cells. Methotrexate treatment increases the expression of lincRNA-p21 and inhibits $\mathrm{NF}-\kappa \mathrm{B}$ activity compared with those in untreated RA patients $(26,27)$. Patients with osteoarthritis (OA) were indicated to have high levels of lncRNA-CIR. Treatment of chondrocytes in vitro with IL-1 $\beta$ and TNF- $\alpha$ significantly increased the expression of IncRNA-CIR compared with that in the controls. Furthermore, silencing of lncRNA-CIR by small interfering RNA reduced the expression of matrix metalloproteinase (MMP)13 and ADAM metallopeptidase with thrombospondin type 1 motif 5 compared with that in the controls. Thus, lncRNA-CIR may act as a potential target in OA therapy $(28,29)$. To examine the functional implications of lncRNAs in GA, the expression profiles of lncRNAs 
A

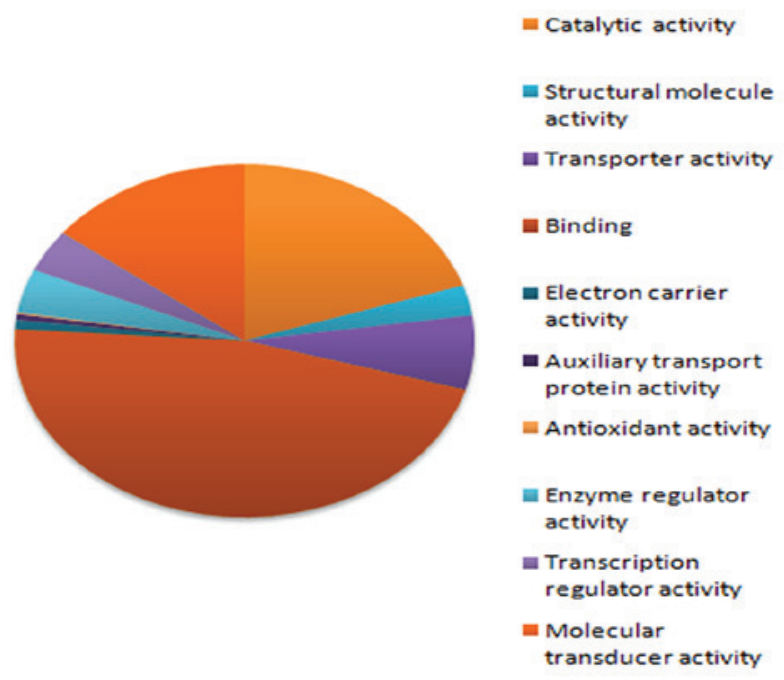

B

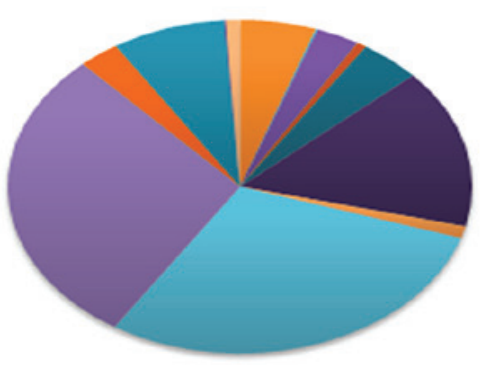

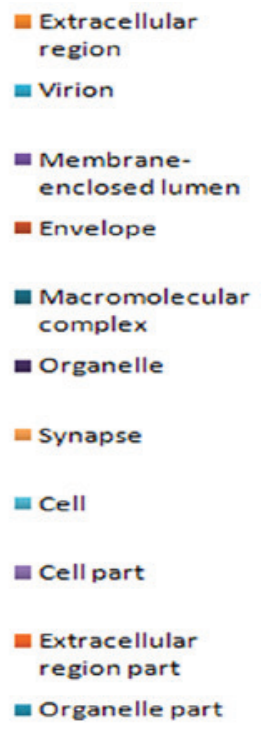

- Virion part

ESynapse part

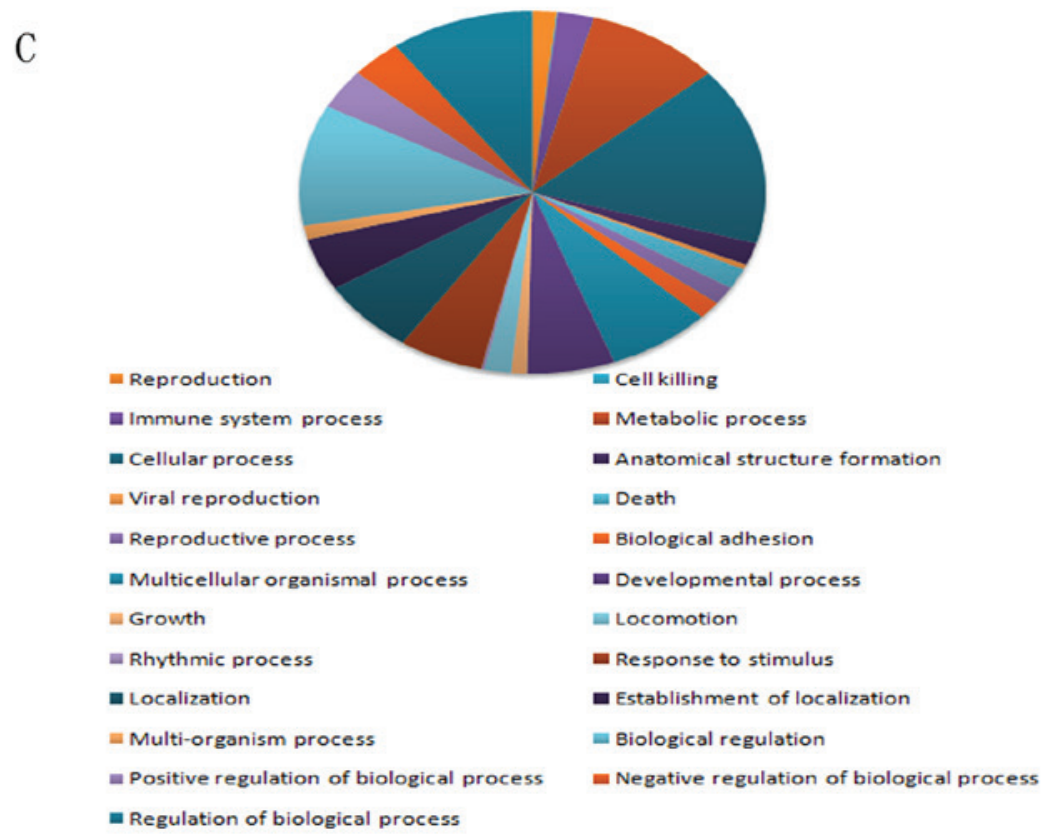

Figure 2. Gene Ontology analysis of the differentially expressed transcripts. (A) Molecular function. (B) Cellular component. (C) Biological process.

in GA patients and in HCs were determined using microarrays.

In the present study, the lncRNA expression profiles in 3 GA patients and $3 \mathrm{HCs}$ comprising 63,431 lncRNAs were assessed by microarray analysis. A total of $875 \operatorname{lncRNAs}$ were significantly upregulated and $940 \mathrm{lncRNAs}$ were significantly downregulated, which was by $>2$-fold. A total of 6 lncRNAs, which were not previously reported by studies on other diseases, were then randomly selected for validation in 60 other GA patients and in paired HCs by RT-qPCR. The results of the RT-qPCR analysis were consistent with those of the microarray analysis. The differential expression of lncRNAs in GA implied that lncRNAs may have a crucial role in the onset and development of GA. However, further validations and functional studies are warranted in the future. In the present study, a significant increase in the expression level of AJ227913 in GA patients compared with that in HCs was identified based on the microarray analysis. The AJ227913 expression levels in GA patients had a close correlation with urea $(\mathrm{r}=0.618, \mathrm{P}<0.05)$, creatinine $(\mathrm{r}=0.382, \mathrm{P}<0.05)$ and cystatin $\mathrm{C}(\mathrm{r}=0.482, \mathrm{P}<0.05)$ levels based on the Spearman correlation analysis. Most circulating uric acid is freely filtered in the kidney. Urea, creatinine, cystatin $\mathrm{C}$ and uric acid are crude indicators of renal filtration and excretory function. However, uric acid is excreted in urine in healthy humans. Uric acid excretion may be impaired by kidney disease, leading to chronic hyperuricaemia. High uric acid levels in the body may lead to glomerular filtration disorders and a decrease in renal tubular secretion. It may increase the risk of gout or gouty nephropathy $(30,31)$. These results suggest that AJ227913 may 


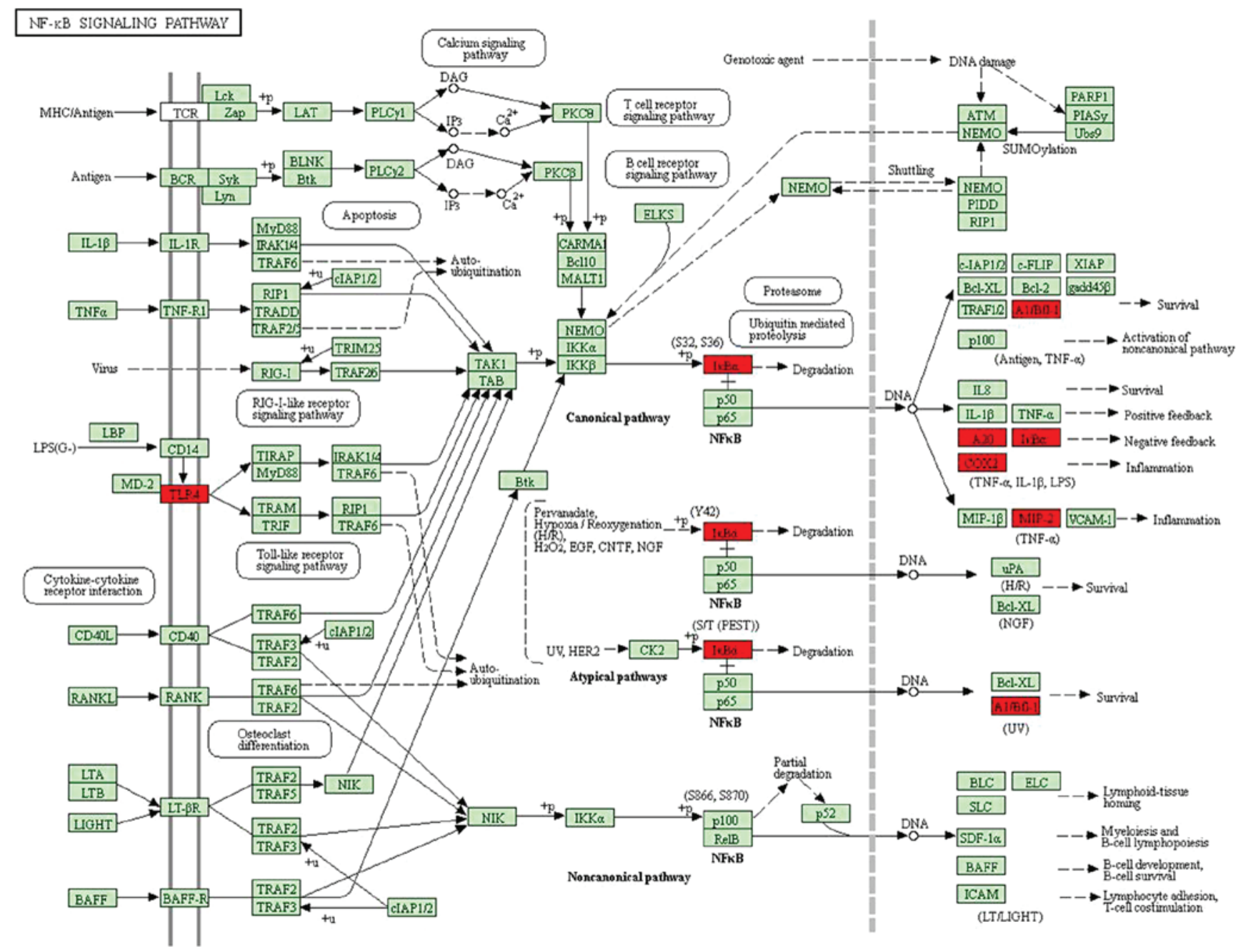

Figure 3. Schematic diagram of the gene category of the NF-kB signaling pathway.

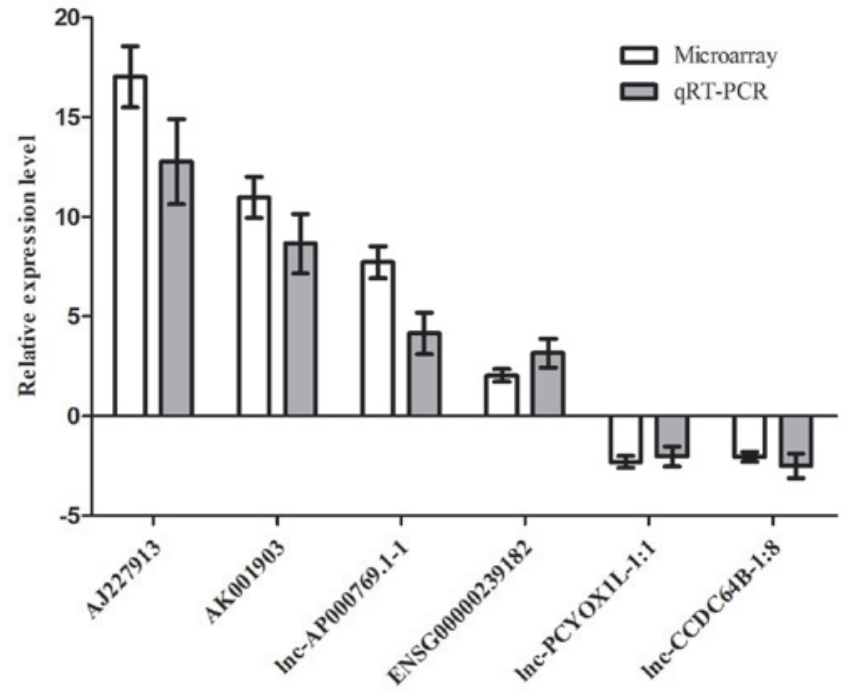

Figure 4. Comparison of the lncRNA expression levels between the microarray and RT-qPCR results. Six differentially expressed lncRNAs were validated by RT-qPCR. The Y-axis represents the log-transformed median fold change (gouty arthritis/healthy controls) in the expression across 60 samples. Data presented as the mean $\pm \mathrm{SD}$. The RT-qPCR results were consistent with those of the microarray data. lncRNA, long non-coding RNA; RT-qPCR, reverse transcription-quantitative PCR. be involved in the dynamic balance of the production and elimination of uric acid in GA patients.

GO and pathway analyses were performed to predict the biological functions of the differentially expressed lncRNAs and potential mechanisms in GA progression. According to this analysis, the mostly highly enriched GO terms by the differentially expressed transcripts were binding, catalytic activity, molecular transducer activity, cellular process, cell and cell part. The GO project is a framework for modelling biology that may be used to describe the functional classification of differentially expressed transcripts. The pathway analysis suggested that the differentially expressed transcripts were associated with 19 pathways. The gene category 'TNF signaling pathway' promotes cell survival and differentiation, as well as immune and inflammatory responses (17). The 'osteoclast differentiation' process is known to be important for bone development and repair (18). In addition, the 'NOD-like receptor signaling pathway' and 'NF- $\kappa \mathrm{B}$ signaling pathway' have been reported to participate in MSU-mediated signaling cascades, which induce inflammasome-dependent gouty inflammation (19). These results have prompted us to investigate the molecular mechanisms of the pathogenesis of gout, which is important for further studies. 
Table IV. KEGG pathway analysis of differentially expressed transcripts.

\begin{tabular}{|c|c|c|c|c|}
\hline Term & Description & Count & P-value & Enrichment score \\
\hline hsa05134 & Legionellosis & 6 & 0.001 & 5.217 \\
\hline hsa04621 & NOD-like receptor signaling pathway & 6 & 0.001 & 5.034 \\
\hline hsa04610 & Complement and coagulation cascades & 7 & 0.001 & 4.238 \\
\hline hsa05150 & Staphylococcus aureus infection & 5 & 0.007 & 4.195 \\
\hline hsa04668 & TNF signaling pathway & 11 & 0.000 & 3.913 \\
\hline hsa05140 & Leishmaniasis & 6 & 0.004 & 3.878 \\
\hline hsa04978 & Mineral absorption & 4 & 0.023 & 3.679 \\
\hline hsa05120 & Epithelial cell signaling in Helicobacter pylori infection & 5 & 0.014 & 3.516 \\
\hline hsa04012 & ErbB signaling pathway & 6 & 0.010 & 3.261 \\
\hline hsa05133 & Pertussis & 5 & 0.020 & 3.188 \\
\hline hsa04064 & $\mathrm{NF}-\kappa \mathrm{B}$ signaling pathway & 10 & 0.013 & 3.085 \\
\hline hsa05131 & Shigellosis & 4 & 0.047 & 2.943 \\
\hline hsa04380 & Osteoclast differentiation & 8 & 0.006 & 2.898 \\
\hline hsa05132 & Salmonella infection & 5 & 0.034 & 2.780 \\
\hline hsa05142 & Chagas disease (American trypanosomiasis) & 6 & 0.021 & 2.759 \\
\hline hsa05161 & Hepatitis B & 7 & 0.033 & 2.293 \\
\hline hsa04024 & cAMP signaling pathway & 9 & 0.023 & 2.163 \\
\hline hsa05205 & Proteoglycans in cancer & 9 & 0.028 & 2.100 \\
\hline hsa05166 & HTLV-I infection & 8 & 0.020 & 2.031 \\
\hline
\end{tabular}

Hsa, Homo sapiens; ErbB, epidermal growth factor; NOD, nucleotide binding oligomerization domain; TNF, tumor necrosis fator; NF- $\kappa \mathrm{B}$, nuclear factor $\kappa \mathrm{B}$; cAMP, cyclic adenosine monophosphate; HTLV-I, human T-lymphotropic virus 1.

Gene expression is a highly complex, regulated process with numerous levels of regulation (epigenetic, transcriptional, post-transcriptional, translational and posttranslational). lncRNAs act as essential regulators of gene expression at the epigenetic level. The results of the 'cis' analysis performed in the present study suggested that AJ227913 may regulate IL-8 expression. IL-8 is a member of the chemokine family and is a key inflammatory mediator, which has an important role in regulating inflammation and immunity in various rheumatic diseases. Significantly higher concentrations of IL-8 were detected in the synovial fluid of RA patients than in those of HCs. IL-8 may stimulate neutrophils to produce cartilage-degrading enzymes, which may lead to joint tissue damage in RA. Inhibiting IL-8 production has been reported to reduce joint damage, which may be a novel target for the treatment of RA (32-34). Previous studies have indicated a marked increase in IL-8 in the exudate from patients with psoriatic arthritis (PsA) compared with that in the controls. An IL-8 monoclonal antibody was approved as a topical treatment for PsA in China. The therapeutic effect of anti-IL-8 is thought to be associated with a decrease in the accumulation of neutrophils and inflammatory cells, leading to the reduction of inflammation in patients with PsA (35-37). These results suggest a pathogenic role for IL-8 in rheumatic diseases. GA is a type of inflammatory arthritis that is caused by the deposition of MSU crystals. Several lines of evidence indicate that IL-8 is an essential mediator of neutrophil-mediated acute inflammation. The levels of IL-8 are increased during the acute and intercritical phases of GA. MSU may stimulate the secretion of IL- 8 by human neutrophils. In rabbits with MSU crystal-induced arthritis, intra-articular injection of anti-IL-8 significantly attenuated crystal-induced joint swelling (38-40). Therefore, it was hypothesized that AJ227913 may regulate IL-8 expression in GA patients, and is thus involved in the pathogenesis of GA. Further research focusing on the roles of AJ227913 may provide a novel strategy for the treatment of GA.

In summary, the present study determined the expression profiles of patients with GA vs. HCs using microarray and identified a collection of differentially expressed lncRNAs. To the best of our knowledge, the present study was the first to examine the lncRNA profiles in GA. The present results may indicate that the differential expression of various IncRNAs has an important role in the development and progression of GA. Further studies will focus on the biological function of lncRNAs involved in GA.

\section{Acknowledgements}

Not applicable.

\section{Funding}

The authors acknowledge the financial support from the Applied Basic Research Program of Sichuan Province (grant no. 2017JY0151), the Science and Technology Support Program of Nanchong (grant nos. NSMC20170436 and 18SXHZ0513) and the Development of Scientific Research Plan of Doctoral Scientific Research Foundation of North Sichuan Medical College (grant no. CBY14-QD-07). 


\section{Availability of data and materials}

The datasets used and/or analyzed during the current study are available from the corresponding author on reasonable request.

\section{Authors' contributions}

$\mathrm{XZ}$ conducted the majority of the experiments and wrote the manuscript. XG and JZ conceived and designed the study. YP and HL performed microarray analysis. CY and JL performed PCR assays. QY, YH and YQ collected blood samples and analyzed the clinical data. All authors read and approved the final manuscript.

\section{Ethics approval and consent to participate}

The present study was approved by the Ethics Committee of the Affiliated Hospital of the North Sichuan Medical College (Nantong, China; IRB: 2015-EA-016). All of the participants who participated in the study provided written informed consent at the time of enrolment.

\section{Patient consent for publication}

Not applicable.

\section{Competing interests}

The authors declare that they have no competing interests.

\section{References}

1. Richette P and Bardin T: Gout. Lancet 375: 318-328, 2010.

2. Kuo CF, Grainge MJ, Zhang W and Doherty M: Global epidemiology of gout: Prevalence, incidence and risk factors. Nat Rev Rheumatol 11: 649-662, 2015.

3. Zhang X, Yang X, Wang M, Li X, Xia Q, Xu S, Xu J, Cai G, Wang L, Xin L, et al: Association between SLC2A9 (GLUT9) gene polymorphisms and gout susceptibility: An updated meta-analysis. Rheumatol Int 36: 1157-1165, 2016.

4. Matsuo H, Takada T, Ichida K, Nakamura T, Nakayama A, Ikebuchi Y, Ito K, Kusanagi Y, Chiba T, Tadokoro S, et al: Common defects of ABCG2, a high-capacity urate exporter, cause gout: A function-based genetic analysis in a Japanese population. Sci Transl Med 1: 5ra11, 2009.

5. Tan PK, Farrar JE, Gaucher EA and Miner JN: Coevolution of URAT1 and uricase during primate evolution: Implications for serum urate homeostasis and gout. Mol Biol Evol 33: 2193-2200, 2016.

6. Pennisi E: Cell biology. Lengthy RNAs earn respect as cellular players. Science 344: 1072, 2014.

7. Wang C, Wang L, Ding Y, Lu X, Zhang G, Yang J, Zheng H, Wang $\mathrm{H}$, Jiang Y and Xu L: LncRNA structural characteristics in epigenetic regulation. Int J Mol Sci 18: E2659, 2017.

8. Saha P, Verma S, Pathak RU and Mishra RK: Long Noncoding RNAs in mammalian development and diseases. Adv Exp Med Biol 1008: 155-198, 2017.

9. Kwok ZH and Tay Y: Long noncoding RNAs: lincs between human health and disease. Biochem Soc Trans 45: 805-812, 2017.

10. Khanna D, Khanna PP, Fitzgerald JD, Singh MK, Bae S, Neogi T, Pillinger MH, Merill J, Lee S, Prakash S, et al: 2012 American college of rheumatology guidelines for management of gout. Part 2: Therapy and anti-inflammatory prophylaxis of acute gouty arthritis. Arthritis Care Res (Hoboken) 64: 1447-1461, 2012.

11. Gomez JA, Wapinski OL, Yang YW, Bureau JF, Gopinath S Monack DM, Chang HY, Brahic M and Kirkegaard K: The NeST Long ncRNA controls microbial susceptibility and epigenetic activation of the interferon-gamma locus. Cell 152: 743-754, 2013.
12. Lai F, Orom UA, Cesaroni M, Beringer M, Taatjes DJ, Blobel GA and Shiekhattar R: Activating RNAs associate with Mediator to enhance chromatin architecture and transcription. Nature 494: 497-501, 2013.

13. Poliseno L, Salmena L, Zhang J, Carver B, Haveman WJ and Pandolfi PP: A coding-independent function of gene and pseudogene mRNAs regulates tumour biology. Nature 465: 1033-1038, 2010 .

14. The Gene Ontology Consortium: Expansion of the Gene Ontology knowledgebase and resources. Nucleic Acids Res 45 (D1): D331-D338, 2017.

15. Kanehisa M, Furumichi M, Tanabe M, Sato Y and Morishima K: KEGG: New perspectives on genomes, pathways, diseases and drugs. Nucleic Acids Res 45: D353-D361, 2017.

16. Schmittgen TD and Livak KJ: Analyzing real-time PCR data by the comparative C(T) method. Nat Protoc 3: 1101-1108, 2008.

17. Kalliolias GD and Ivashkiv LB: TNF biology, pathogenic mechanisms and emerging therapeutic strategies. Nat Rev Rheumatol 12: 49-62, 2016.

18. Shapiro F: Bone development and its relation to fracture repair. The role of mesenchymal osteoblasts and surface osteoblasts. Eur Cell Mater 15: 53-76, 2008.

19. Qing YF, Zhang QB and Zhou JG: Innate immunity functional gene polymorphisms and gout susceptibility. Gene 524: 412-414, 2013.

20. Mattick JS: Non-coding RNAs: The architects of eukaryotic complexity. EMBO Rep 2: 986-991, 2001.

21. Stachurska A, Zorro MM, van der Sijde MR and Withoff S: Small and long regulatory RNAs in the immune system and immune diseases. Front Immunol 5: 513, 2014.

22. Luo Q, Li X, Xu C, Zeng L, Ye J, Guo Y, Huang Z and Li J: Integrative analysis of long non-coding RNAs and messenger RNA expression profiles in systemic lupus erythematosus. Mol Med Rep 17: 3489-3496, 2018.

23. Li LJ, Zhao W, Tao SS, Li J, Xu SZ, Wang JB, Leng RX, Fan YG, Pan HF and Ye DQ: Comprehensive long non-coding RNA expression profiling reveals their potential roles in systemic lupus erythematosus. Cell Immunol 319: 17-27, 2017.

24. Wang P, Xue Y, Han Y, Lin L, Wu C, Xu S, Jiang Z, Xu J, Liu Q and Cao X: The STAT3-binding long noncoding RNA lnc-DC controls human dendritic cell differentiation. Science 344: 310-313, 2014.

25. Wu GC, Li J, Leng RX, Li XP, Li XM, Wang DG, Pan HF and Ye DQ: Identification of long non-coding RNAs GAS5, linc0597 and lnc-DC in plasma as novel biomarkers for systemic lupus erythematosus. Oncotarget 8: 23650-23663, 2017.

26. Pan F, Zhu L, Lv H and Pei C: Quercetin promotes the apoptosis

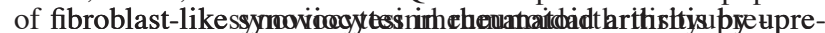
gulating lncRNA MALAT1. Int J Mol Med 38: 1507-1514, 2016.

27. Spurlock CF III, Tossberg JT, Matlock BK, Olsen NJ and Aune TM: Methotrexate inhibits NF- $\kappa \mathrm{B}$ activity via long intergenic (noncoding) RNA-p21 induction. Arthritis Rheumatol 66: 2947-2957, 2014.

28. Liu Q, Zhang X, Dai L, Hu X, Zhu J, Li L, Zhou C and Ao Y: Long noncoding RNA related to cartilage injury promotes chondrocyte extracellular matrix degradation in osteoarthritis. Arthritis Rheumatol 66: 969-978, 2014.

29. Wang CL, Peng JP and Chen XD: LncRNA-CIR promotes articular cartilage degeneration in osteoarthritis by regulating autophagy. Biochem Biophys Res Commun 505: 692-698, 2018.

30. Mount DB: The kidney in hyperuricemia and gout. Curr Opin Nephrol Hypertens 22: 216-223, 2013.

31. Prasad Sah OS and Qing YX: Associations between hyperuricemia and chronic kidney disease: A Review. Nephrourol Mon 7: e27233, 2015.

32. Georganas C, Liu H, Perlman H, Hoffmann A, Thimmapaya B and Pope RM: Regulation of IL-6 and IL-8 expression in rheumatoid arthritis synovial fibroblasts: The dominant role for NF-kappa B but not C/EBP beta or c-Jun. J Immunol 165: 7199-7206, 2000.

33. De Benedetti F, Pignatti P, Bernasconi S, Gerloni V, Matsushima K, Caporali R, Montecucco CM, Sozzani S, Fantini $\mathrm{F}$ and Martini A: Interleukin 8 and monocyte chemoattractant protein-1 in patients with juvenile rheumatoid arthritis. Relation to onset types, disease activity, and synovial fluid leukocytes. J Rheumatol 26: 425-431, 1999.

34. Lun SW, Wong CK, Tam LS, Li EK and Lam CW: Decreased ex vivo produxtion of TNF-alpha and IL- 8 by peripheral blood cells of patients with rheumatoid arthritis after infliximab therapy. Int Immuno pharmacol 7: 1668-1677, 2007. 
35. König A, Krenn V, Gillitzer R, Glöckner J, Janssen E, Gohlke F, Eulert J and Müller-Hermelink HK: Inflammatory infiltrate and interleukin-8 expression in the synovium of psoriatic arthritis-an immunohistochemical and mRNA analysis. Rheumatol Int 17: 159-168, 1997.

36. Biasi D, Carletto A, Caramaschi P, Bellavite P, Maleknia T, Scambi C, Favalli N and Bambara LM: Neutrophil functions and IL-8 in psoriatic arthritis and in cutaneous psoriasis Inflammation 22: 533-543, 1998.

37. Tsai YC and Tsai TF: Anti-interleukin and interleukin therapies for psoriasis: Current evidence and clinical usefulness. Ther Ady Musculoskelet Dis 9: 277-294, 2017.

38. Kienhorst LB, van Lochem E, Kievit W, Dalbeth N, Merriman ME, Phipps-Green A, Loof A, van Heerde W, Vermeulen S, Stamp LK, et al: Gout is a chronic inflammatory disease in which high levels of interleukin-8 (CXCL8), myeloid-related protein 8/myeloid-related protein 14 complex, and an altered proteome are associated with diabetes mellitus and cardiovascular disease. Arthritis Rheumatol 67: 3303-3313, 2015.
39. Hachicha M, Naccache PH and McColl SR: Inflammatory microcrystals differentially regulate the secretion of macrophage inflammatory protein 1 and interleukin 8 by human neutrophils: A possible mechanism of neutrophil recruitment to sites of inflammation in synovitis. J Exp Med 182: 2019-2025, 1995.

40. Nishimura A, Akahoshi T, Takahashi M, Takagishi K, Itoman M, Kondo H, Takahashi Y, Yokoi K, Mukaida N and Matsushima K: Attenuation of monosodium urate crystal-induced arthritis in rabbits by a neutralizing antibody against interleukin-8. J Leukoc Biol 62: 444-449, 1997.

(c) (1) (9) This work is licensed under a Creative Commons Attribution-NonCommercial-NoDerivatives 4.0 International (CC BY-NC-ND 4.0) License. 